\title{
Dal Senato all'Acquario AIRP non si ferma mai
}

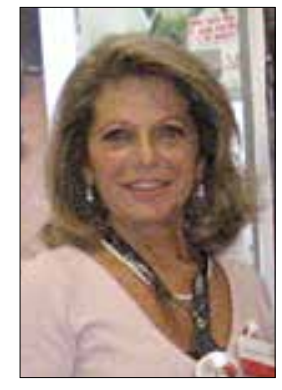

Luisa Sternfeld Pavia

\section{Cari amici,}

Permettetemi di cominciare con un grande ringraziamento a tutti voi. I vostri contributi, devoluti con il $5 \times 1000$, sono stati e continuano a essere fondamentali per proseguire il lavoro di ricerca sostenuto da AIRP.

Come sapete, nel 2015, grazie alle vostre donazioni, è stato avviato, presso I'IRCCS Ospedale San Raffaele, lo studio per il trial sperimentale sull'uomo per verificare, in primis, la tollerabilità della molecola 2-deossi-glucosio (2DG) sull'uomo nella regressione e nella riduzione delle cisti renali. La Dr.ssa Alessandra Boletta e la sua equipe hanno ottenuto ottimi risultati e siamo molto felici di poter annunciare che AIRP è in grado di aumentare per il 2016 l'importo per il sostegno a questa ricerca clinica.

C'è un secondo importante traguardo che si è potuto raggiungere grazie alle vostre donazioni: è il test genetico per l'analisi dei geni PKD1 e PKD2 e ARPKD con il metodo NGS, che è oggi possibile effettuare nel laboratorio di Genetica Medica degli Spedali Civili di Brescia. Questo test rappresenta una vera e propria conquista per la qualità della vita dei pazienti e delle loro famiglie e, anche in questo caso, è stato decisivo il contributo di AIRP, quindi il vostro.

Tengo fortemente a sottolineare che, senza il vostro aiuto, nulla di tutto ciò avrebbe potuto realizzarsi. Ecco perché vi invito a continuare a donare ad AIRP onlus per sostenere insieme la ricerca sul Rene Policistico e dare, ogni giorno, nuova speranza a chi lotta contro i dolorosi effetti di questa patologia.

La partecipazione diretta di AIRP al mondo della ricerca e della clinica è dimostrata, oltre che nel caso dell'Ospedale San Raffaele di Milano, anche nel Centro di Eccellenza di Montichiari (BS), dove i pazienti affetti da rene policistico

Accepted: July 5, 2016

Published online: July 18, 2016

Indirizzo per la corrispondenza:

Dr.ssa Luisa Sternfeld Pavia

AIRP Associazione Italiana Rene Policistico onlus

Via A. Bazzini 2

20131 Milano

luisa.sternfeld.airp@renepolicistico.it trovano un team multi-specialistico di altissimo livello.

Come spiega il Prof. Francesco Scolari: "Se, in passato, i nefrologi consideravano il Rene Policistico una patologia dal decorso immutabile, le tecniche moderne di biologia cellulare offrono una nuova prospettiva per il futuro: la possibilità di interferire con la progressiva crescita delle cisti. Ciò richiederà investimenti sempre maggiori nella ricerca clinica e delle figure che possano rispondere al carattere complesso della patologia renale policistica". Attualmente, I'Ambulatorio di Montichiari vede la collaborazione di nefrologi, radiologi, cardiologi, urologi, ma non solo: da circa tre anni, è stato incluso nel team anche un medico genetista clinico. Questo consente un approccio multi-specialistico alla malattia, oltre alla possibilità di utilizzare strumentazioni tecnologiche molto sofisticate per la diagnosi anche prenatale. Ebbene, l'ambizioso percorso di crescita di questo Ambulatorio ha trovato, nel sostegno di AIRP, un alleato fondamentale e ne siamo fieri.

Il nostro impegno è altrettanto intenso sul fronte delle istituzioni. AIRP ha, infatti, incontrato, a fine Aprile, la senatrice Laura Bianconi, Autrice di un'interrogazione parlamentare sulle intenzioni del Governo riguardo ai pazienti con ADPKD. La seduta si è, poi, svolta il 21 Giugno e ha affrontato diverse tematiche che ci riguardano direttamente.

In primo luogo, ricordiamo che esiste, presso il Ministero della Salute, un Tavolo di lavoro multidisciplinare dedicato alla "Malattia renale cronica".

Nell'ambito dell'aggiornamento complessivo dei Livelli essenziali di assistenza (LEA), previsto dal Patto per la salute 2014/2016, è stato proposto l'inserimento del "rene policistico autosomico dominante" nell'elenco delle patologie croniche e invalidanti.

Questo consentirà ai pazienti di usufruire, in esenzione, delle prestazioni specialistiche ambulatoriali appropriate per il monitoraggio della malattia e per la prevenzione degli eventuali aggravamenti.

Il gruppo di lavoro del Senato ha affrontato anche il tema del Tolvaptan, per il quale era stato chiesto all'AIFA I'inserimento in classe $\mathrm{H}$ (farmaci essenziali e farmaci per malattie croniche) e l'assegnazione del regime di fornitura RNRL (ricetta non ripetibile limitativa). L'Agenzia del farmaco, tuttavia, ha deciso, per ora, di mantenere il Tolvaptan in classe $C$, in attesa di vagliare risultati consolidati sugli effetti a lungo termine del trattamento su "endpoint" clinicamente rilevanti.

Per il momento, quindi, accettiamo con soddisfazione il fatto che la nostra patologia sia oggetto di discussione da parte delle istituzioni e proseguiamo la nostra battaglia per 
ottenere attenzione e cure adeguate.

Per quanto riguarda i prossimi mesi, vi anticipo tre importanti eventi.

Giornata all'Acquario di Genova in programma per Sabato 3 Settembre 2016. Sarà una giornata interamente dedicata ai nostri piccoli pazienti e alle loro famiglie, da vivere insieme in una delle attrazioni più affascinanti per piccoli e grandi (Fig. 1).

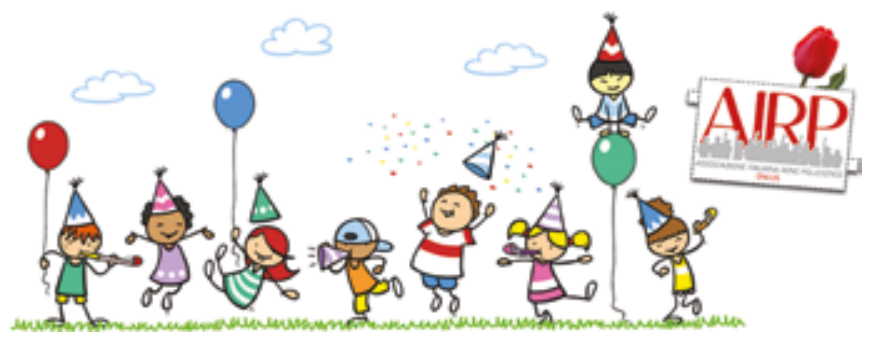

Fig. 1 - La giornata all'Acquario di Genova.

> Giovedì 29 Settembre 2016 a Fermo si svolgerà l'8 $8^{\circ}$ Gran Galà della Solidarietà, una cena solidale promossa da AIRP e organizzata da Alberta e Rosita, due volontarie molto attive, con lo scopo di raccogliere fondi per la Ricerca scientifica e per le attività di AIRP (Fig. 2).

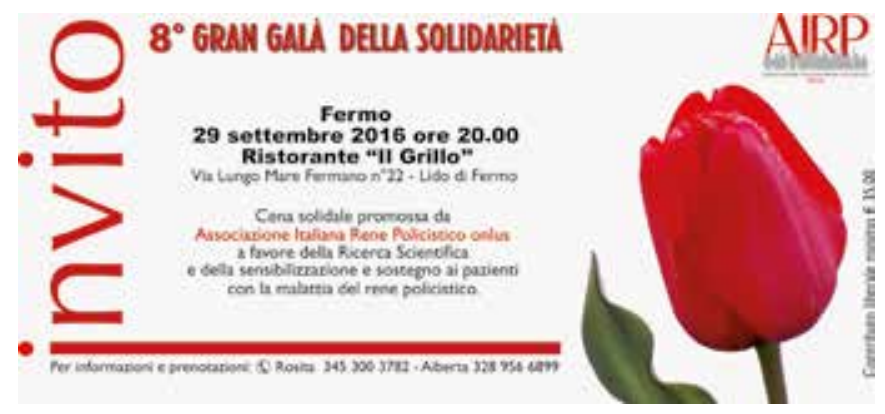

Fig. 2 - Gran Galà della Solidarietà.
Sabato 3 Dicembre 2016 si svolgerà un meeting all'Università di Brescia, dal titolo "Rene Policistico: il futuro inizia adesso - Percorso di ricerca, diagnosi e accesso alle cure - "ll dolore è ancor più dolore se tace" (Giovanni Pascoli)"(Fig. 3).

Direttore Scientifico: Prof. Francesco Scolari.

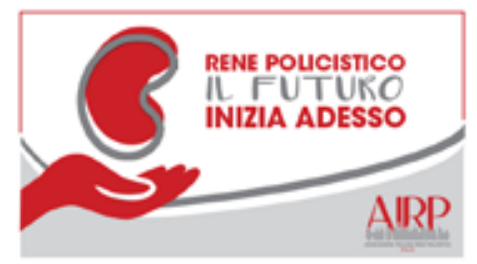

Rene Policistico: il futuro inizia adesso Percorso di ricerca, diagnosi e accesso alle cure all dolore ò ancor piò dolore se locos (Giovanri Pascoli)

\section{3 dicembre 2016}

\section{Aula Magna}

Universita degli Studi di Brescio Focolla di Medicina e Chirurgia

DIRETTORE SCIENTIFCO - Prot. Francesco Scolan SEcreteru SCIENTIFICA - Dr.ssa Cloudic lzzi e AIRP onlus Associozione aliano Rene Policiatiog onlus SEGRETERLA ORGLNIzZATIVA - Aleno Congressi - Vio Pontin 1050018 Scondicol (Fivence) atonocongressioctinocongressi \&

Fig. 3 - Meeting di Brescia.

Sul nostro sito www.renepolicistico.it, nella sezione "Eventi", troverete i programmi di tutti gli eventi e le relative modalità di partecipazione.

In attesa, quindi, di incontrarvi in una delle prossime iniziative, auguro buona estate a tutti voi!

Luisa Sternfeld Pavia 